Polymer Journal, Vol. 3, No. 4, pp 526-530 (1972)

\title{
Polymerization of $\beta$-Acetoxypropionaldehyde
}

\author{
Hiroshi Sumitomo, Kazukiyo Kobayashi, and \\ Yoshinobu YAMADA \\ Faculty of Agriculture, Nagoya University, \\ Chikusa, Nagoya 464, Japan. \\ (Received December 13, 1971)
}

\begin{abstract}
High crystalline and high molecular weight poly[(acetoxyethyl)oxymethylene] was prepared from $\beta$-acetoxypropionaldehyde with use of triethylaluminum and diethylaluminum chloride at low temperature. It was also established by infrared, NMR, and X-ray analyses that even protonic acid such as sulfuric acid could produce the crystalline, isotactic poly[(acetoxyethyl)oxymethylene] at $-78^{\circ} \mathrm{C}$. It is suggested that the polar acetoxy group participates in the steric control of polymerization and the mechanism is presumed.
\end{abstract}

KEY WORDS $\beta$-Acetoxypropionaldehyde / Stereospecific Polymerization / Crystalline Poly[(acetoxyethyl)oxymethylene] / Polar Substituent / Protonic Acid / Organoaluminum /

As part of the studies on polar-substituted poly(oxymethylene)s, ${ }^{1-3}$ the present paper describes an attempt of polymerizing $\beta$-acetoxypropionaldehyde at low temperature and of characterizing the resulting polymer. It was found that crystalline isotactic poly[(acetoxyethyl)oxymethylene] is produced by organoaluminum and protonic acid. It is noticeable that a protonic acid such as sulfuric acid could produce the crystalline polymer and attention should be drawn to the role of polar substituents in the stereospecific polymerization.

\section{EXPERIMENTAL}

$\beta$-Acetoxypropionaldehyde was prepared from acrolein and acetic acid according to the method of Fischer, et al. ${ }^{4}$ and was purified by fractional distillation just before use: bp $60-63^{\circ} \mathrm{C}(7 \mathrm{~mm})$ $\left[\right.$ lit. $\left.^{4} 65-70^{\circ} \mathrm{C}(10 \mathrm{~mm})\right] ; d_{4}^{20} 1.0929$. Elemental analysis was made for the 2,4-dinitrophenylhydrazone of the aldehyde.

Anal. Calcd. for $\mathrm{C}_{11} \mathrm{H}_{12} \mathrm{~N}_{4} \mathrm{O}_{6}: \mathrm{C}, 44.59 ; \mathrm{H}$, 4.08; N, 18.92; $M W, 296$. Found: C, 44.15; H, 4.26; N, 18.60; $M W, 287$ (determined by vapor pressure osmometry in benzene at $37^{\circ} \mathrm{C}$ ).

The purity of the monomer was also checked by gas chromatography and NMR spectroscopy.

Triethylaluminum and diethylaluminum chloride (both from Ethyl Corp.) were distilled under reduced pressure and stored in sealed glass ampules until use. Sulfuric acid (sp gr, ca. 1. 84) and $35 \%$ hydrochloric acid were standard reagent grade chemicals. Polymerization solvents were purified in the usual ways.

Polymerization procedure was the same as already reported..$^{1-3}$ In a dry box filled with dry nitrogen, the monomer diluted with a solvent was gradually added to a catalyst solution in a glass ampule which as previously cooled below $-80^{\circ} \mathrm{C}$ with liquid nitrogen. After the solution was degassed, the ampule was sealed and allowed to stand in a Dewar flask filled with a refrigerant $\left(-78\right.$ and $\left.-100^{\circ} \mathrm{C}\right)$ or in an electronic cooling thermostat $\left(-21\right.$ and $\left.-45^{\circ} \mathrm{C}\right)$. Polymerization was terminated and the product was washed thoroughly by adding a large amount of cold methanol; the precipitated polymer was collected on a glass filter. When the polymerization was initiated by sulfuric acid, cold methanol-pyridine mixture was used for termination and the product was separated by centrifugation.

Infrared spectra were measured with a 215 Hitachi grating infrared spectrophotometer and NMR spectra were recorded on a Japan Electron Optics Lab. Model JNM-4H-100 high resolution spectrometer at $100 \mathrm{MHz}$. The spectra were measured in $\mathrm{CDCl}_{3}$ at a concentration of $10 \%$ at room temperature using tetramethylsilane as an internal standard. X-ray diffraction was performed employing a Rigaku Denki Cat. No. 2030/R 
Geigerflex diffractometer. Copper $\mathrm{K}_{\alpha}$ radiation through a Ni filter was used. The DTA measurement was made with a Rigaku Denki Cat. No. 8001 at a heating rate of $5^{\circ} \mathrm{C} / \mathrm{min}$.

\section{RESULTS AND DISCUSSION}

Table I summarizes the results of polymerization of $\beta$-acetoxypropionaldehyde. White powdery polymer was obtained with triethylaluminum and diethylaluminum chloride in the temperature range of $-100^{\circ} \mathrm{C}$ to $-21^{\circ} \mathrm{C}$. The highest yield was attained at $-78^{\circ} \mathrm{C}$. The polymerization system at $-78^{\circ} \mathrm{C}$ was observed to set within one day and subsequently the yield remained nearly constant. The yield is found to decrease in the order of methylene chloride $>$ THF $>$ toluene used as solvents. The polymer is insoluble in common organic solvents and slightly soluble in dimethyl sulfoxide. Melting point determined by differential thermal analysis is about $160^{\circ} \mathrm{C}$.

White solid mass product could be obtained by sulfuric acid catalyst at $-78^{\circ} \mathrm{C}$. It was so unstable in air at room temperature that it had promptly to be endcapped by treating its chloroform solution with acetic anhydride and pyridine at $90-95^{\circ} \mathrm{C}$ for $1 \mathrm{hr}$. This polymer was soluble in methylene chloride and chloroform but insoluble in other organic solvents. The intrinsic viscosity $[\eta](100 \mathrm{ml} / \mathrm{g})$ measured using an Ostwald viscometer at $25^{\circ} \mathrm{C}$ was 0.064 in methylene chloride and 0.072 in chloroform. The DTA curve showed an endotherm peaking at about $80^{\circ} \mathrm{C}$.

The infrared spectra of the polymers obtained above are shown in Figure 1. While the disappearance of $\nu_{\mathrm{C}=\mathrm{O}}$ of the aldehyde group at $\mathbf{1 7 2 5}$ $\mathrm{cm}^{-1}$ was not clearly detectable owing to the presence of the ester-carbonyl group at 1740 $\mathrm{cm}^{-1}$, the $\mathrm{C}-\mathrm{H}$ stretching vibration of the aldehyde disappeared at $2750 \mathrm{~cm}^{-1}$ and the acetal linkage absorptions characteristic of poly(oxymethylene)s occurred in the region of 900-1200 $\mathrm{cm}^{-1}$. This is also reflected in the NMR spectra represented in Figure 2 where triplet signal of the acetal methine proton appears at a $\tau$ value

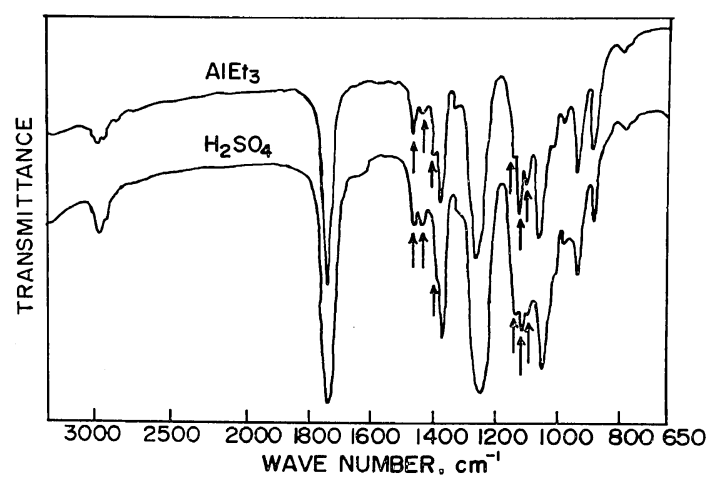

Figure 1. Infrared spectra of poly[(acetoxyethyl)oxymethylene] prepared by triethylaluminum and by sulfuric acid.

Table I. Polymerization of $\beta$-acetoxypropionaldehyde

\begin{tabular}{|c|c|c|c|c|c|c|c|c|}
\hline $\begin{array}{c}\text { Monomer, } \\
\mathrm{g}\end{array}$ & & $\begin{array}{l}\text { Catalyst, mol } \% \\
\text { to monomer }\end{array}$ & Solvent, & $\mathrm{g}$ & $\underset{{ }^{\circ} \mathrm{C}}{\mathrm{Temp}}$ & $\underset{\mathrm{hr}}{\mathrm{Time}}$ & $\underset{\%}{\text { Yield, }}$ & Product \\
\hline 5 & $\mathrm{AlEt}_{3}$ & 2 & Methylene chloride & 15 & -100 & 24 & 11.8 & Powdery polymer \\
\hline$" \prime$ & " & " & " & " & -78 & 10 & 38.3 & " \\
\hline " & $" \prime$ & $" 1$ & " & " & $" \prime$ & 24 & 57.6 & " \\
\hline " & $" \prime$ & " & " & " & " & 48 & 57.3 & " \\
\hline " & $" \prime$ & 3 & " & " & $" \prime$ & 24 & 71.5 & " \\
\hline " & " & 2 & $" \prime$ & " & -45 & "I & 2.6 & " \\
\hline " & $" \prime$ & " & " & " & -21 & " & trace & " \\
\hline " & $" \prime$ & $" \prime$ & THF & " & -78 & $" \prime$ & 32.4 & " \\
\hline " & " & $" \prime$ & Toluene & $" \prime$ & $" \prime$ & " & 30.4 & " \\
\hline " & $\mathrm{AlEt}_{2} \mathrm{Cl}$ & $" \prime$ & Methylene chloride & $" \prime$ & " & "I & 20.5 & " \\
\hline$" \prime$ & $\mathrm{H}_{2} \mathrm{SO}_{4}$ & 0.1 & " & 5 & $" \prime$ & " & 55.1 & Solid mass polymer \\
\hline$" \prime$ & " & 0.2 & "I & $" \prime$ & 0 & " & 31.4 & Cyclic trimer \\
\hline " & $\mathrm{BF}_{3} \mathrm{OEt}_{2}$ & 2 & " & " & -78 & 48 & 21.3 & " \\
\hline$" \prime$ & $\mathrm{HCl}$ & 0.3 & $" \prime$ & $" 1$ & " & 24 & 0 & - \\
\hline 7 & "I. & 0.4 & - & 0 & 25 & 48 & 52.9 & Cyclic trimer \\
\hline
\end{tabular}




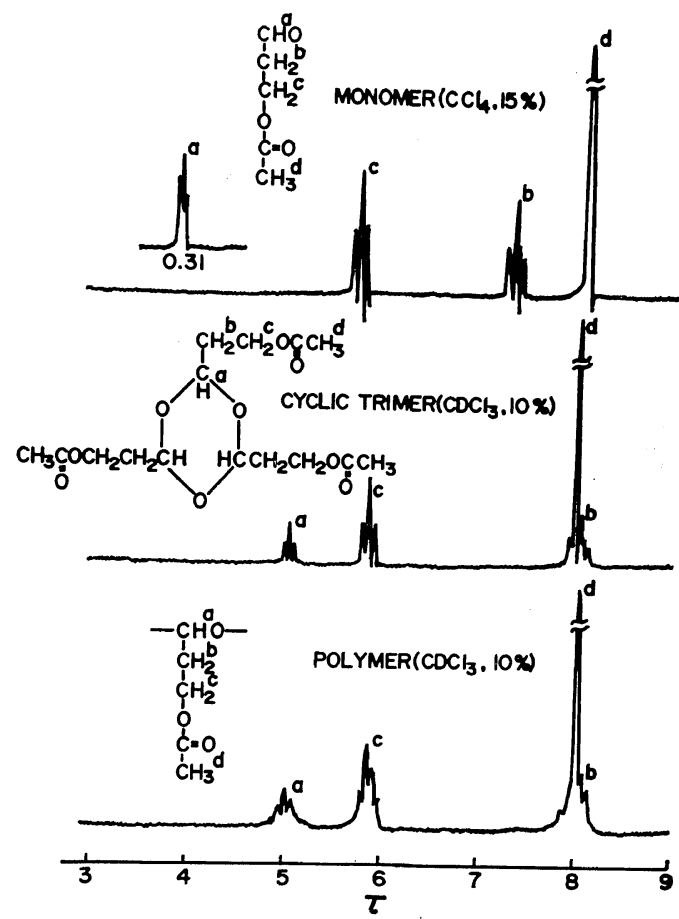

Figure 2. NMR spectra of $\beta$-acetoxypropionaldehyde (in $\mathrm{CCl}_{4}$, at $15 \%$ ), cyclic trimer, and polymer prepared by sulfuric acid $\left(\mathrm{CDCl}_{3}, 10 \%\right)$.

of 5.01 instead of that of the aldehyde methine proton $(0.31 \tau)$.

Treatment of the polymer with an aqueous acid solution of 2,4-dinitrophenylhydrazine gave the same 2,4-dinitrophenylhydrazone as that obtained from the monomer. The results of elementary analysis of the polymers were as follows:

Anal. Found for the polymer prepared with triethylaluminum: $\mathrm{C}, 51.34 ; \mathrm{H}, 6.78$; with sulfuric acid: C, 51.82; $\mathrm{H}, 6.83$. Calcd. for $\left(\mathrm{C}_{5} \mathrm{H}_{8} \mathrm{O}_{3}\right)_{n}$ : C, 51.72; H, 6.94.

These data indicate that $\beta$-acetoxypropionaldehyde was converted through the opening of the aldehyde group to poly[(acetoxyethyl)oxymethylene].

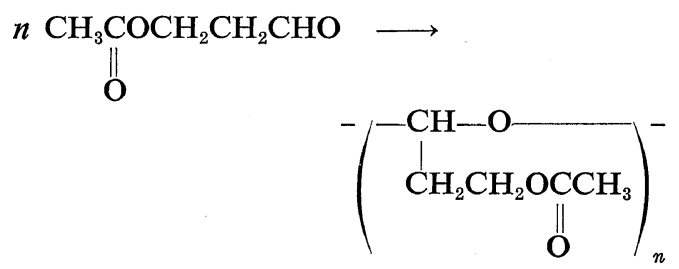

The cyclic trimer of $\beta$-acetoxypropionaldehyde $(2,4,6$-tris $(\beta$-acetoxyethyl)-s-trioxane) was prepared by treating the monomer with several drops of conc. hydrochloric acid at room temperature for two days. After the unreacted monomer had distilled off under reduced pressure, the product was recrystallized from water containing a small amount of ethanol. White needle crystal formed was soluble in common organic solvents: Yield, 53\%; mp 59.5-60 ${ }^{\circ} \mathrm{C}$.

Anal. Calcd. for $\left(\mathrm{C}_{5} \mathrm{H}_{8} \mathrm{O}_{3}\right)_{3}: \mathrm{C}, 51.72 ; \mathrm{H}$, 6.94; $M W, 348$. Found: $\mathrm{C}, 51.49 ; \mathrm{H}, 6.97$; $M W$ (VPO), 343.

The cyclic trimer was also obtained with sulfuric acid at $0^{\circ} \mathrm{C}$ and with boron trifluoride etherate at $-78^{\circ} \mathrm{C}$.

$\mathrm{X}$-ray diffraction diagrams of the polymers are given in Figure 3. Crystalline reflections

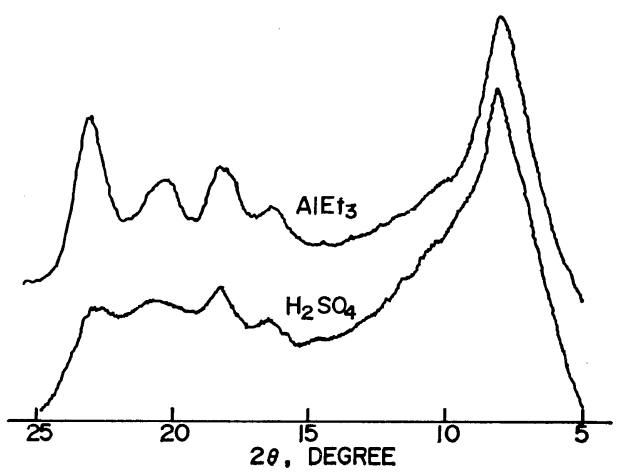

Figure 3. X-ray diffraction diagrams of poly[(acetoxyethyl)oxymethylene] prepared by triethylaluminum and by sulfuric acid.

appear at $2 \theta$ degrees of $8.2^{\circ}, 16.5^{\circ}, 18.1^{\circ}, 20.1^{\circ}$, and $23.1^{\circ}$. The maximum interplanar spacing is calculated to be $10.8 \AA$. Although in the extended state acetoxyethyl group is apparently longer than butyl group, the calculated spacing is smaller than that $(11.8 \mathrm{~A})$ for poly[( $n$-butyl)oxymethylene]. ${ }^{5}$ The spacings are assumed to be the distance between the helix axes in the crystal structure. So poly[(acetoxyethyl)oxymethylene] chains may be more compactly packed than those of poly[( $n$-butyl)oxymethylene]. The same observation was made in the $\mathrm{X}$-ray study of crystalline poly[(cyanoethyl)oxymethylene $].{ }^{1}$ Both acetoxy and cyano groups are so strong polar groups 
that some inter- and/or intra-molecular interactions through the polar side group may be considered to arise.

The amorphous polymer could not be separated by fractional extraction from the polymer prepared by triethylaluminum. It is remarkable that the soluble poly[(acetoxyethyl)oxymethylene] of rather low molecular weight prepared by sulfuric acid exhibits the characteristic crystalline diffraction patterns, although its crystallinity seems slightly lower than that prepared by triethyaluminum. In an earlier paper ${ }^{2}$ the degree of crystallinity of poly[(cyanoethyl)oxymethylene] was estimated from the crystalline- and amorphous bands in the infrared spectra. In the poly(oxymethylene) derivatives such as polyacetaldehyde $^{6}$ and poly[(carbomethoxyethyl)oxymethylene], ${ }^{7}$ the amorphous bands could be clearly distinguished from the crystalline ones. In the present investigation, there appear slight differences between the two IR spectra, as indicated by the arrows in Figure 1, but both spectra can be said to be essentially identical. It may be concluded that sulfuric acid also yields crystalline poly[(acetoxyehtyl)oxymethylene], though it is not as effective as triethylaluminum.

High-resolution NMR spectroscopy was successfully employed in the determination of the configurations of amorphous polyacetaldehyde..$^{8-10}$ NMR study of crystalline polyoxymethylene derivatives, however, has not been properly worked out yet because of the lack of suitable solvents. Crystalline poly[(acetoxyethyl)oxymethylene] prepared by sulfuric acid at $-78^{\circ} \mathrm{C}$ can be dissolved sufficiently in chloroform to succeed in the NMR measurement. The spectrum is shown in Figure 2 , together with that of the cyclic trimer. The cyclic trimer of aldehyde has been considered to be applicable to a model compound for the configurational study of poly(oxymethylene)s. The spectrum of poly[(acetoxyethyl)oxymethylene] resembles that of the cyclic trimer closely. Although the methylene protons at the $\alpha$-position to the main chain overlap on the methoxy protons, the methine proton exhibits a rather wellresolved triplet signal. The chemical shift at $\tau$ 5.01 for the poly(oxymethylene) chain is very close to that for the cyclic trimer ( $\tau$ 5.00).
Jungnickel ${ }^{11}$ indicated that the substituted trioxanes tend to be in the all-cis configuration as follows,

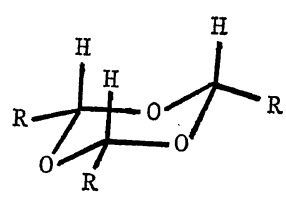

All methine protons, which are axial to the ring, are in quite the same environment and assumed to correspond to the isotactic triad methine in the poly(oxymethylene) chain. Goodman, et al., ${ }^{8}$ confirmed that the backbone chain of amorphous polyacetaldehyde is composed of heterotactic and isotactic triad sequences. In general higher isotactic content seems to be one of the characteristics of amorphous polyoxymethylenes. Crystalline poly(oxymethylene) derivatives such as those from acetaldehyde, ${ }^{12}$ chloral, ${ }^{13}$ and $\beta$-cyanopropionaldehyde ${ }^{2}$ were demonstrated also from $\mathrm{X}$ ray analysis to possess an isotactic configuration. It may be concluded that crystalline poly[(acetoxyethyl)oxymethylene] consists of isotactic sequences.

In the cationic polymerization of vinyl compounds, it has been known that only limted combinations of monomer and initiator lead to stereospecific polymerization. ${ }^{14}$ The cases of alkyl vinyl ethers and $o$-methoxystyrene having polar groups attached to olefinic double bond are the examples, where Lewis acids such as $\mathrm{BF}_{3} \mathrm{OEt}_{2}$ and $\mathrm{AlEtCl}_{2}$ are used as initiators in non-polar solvents at low temperature. The heterogeneous complex of sulfuric acid with metal sulfate was also effective, ${ }^{15}$. but sulfuric acid itself has not yet worked stereochemically. On the other hand, in spite of the inherent liability of acetaldehyde to convert to isotactic sequence, paraldehyde or amorphous polyacetaldehyde was formed with various protonic acids including sulfuric acid at low temperature. So it is quite noticeable that crystalline isotactic poly[(acetoxyethyl)oxymethylene] was prepared here by a protonic acid.

One of the striking features in the polymerization of aldehyde different from vinyl monomers is the contribution of the high polarization of 
the aldehyde group. In the present monomer, not only the aldehyde but also the acetoxy group is highly polar and may participate in the stereospecific control of the polymerization. The latter group might interact with the propagating carbonium ion or with the counter anion. Such an interaction would control the direction of the attack of the approaching monomer on the propagating chain end.

Goodman, et al. ${ }^{8}$ suggested that acetaldehyde may exist in a dimeric form under polymerization condition leading to a preferred incorporation of mesodimeric unit into the propagating chain end. $\beta$-Acetoxypropionaldehyde exhibits rather high boiling point, density, and refractive index, compared with the monofunctional analogues of the similar molecular weight such as heptanal, butyl acetate, and heptanol. It may suggest that the monomer molecules associate through the polar groups to form some molecular aggregate. Such an aggregate might be possible to be oriented to give the isotactic sequence configuration.

The mechanisms mentioned above are only speculative but reasonable by analogy with those proposed for vinyl ethers and acetaldehyde. More detailed experiments are now performing from the various points of view.

\section{REFERENCES}

1. H. Sumitomo and K. Kobayashi, J. Polym. Sci.
Part A-1, 5, 2247 (1967) and the related and following papers.

2. K. Kobayashi and H. Sumitoms, ibid., Part A-1, 2, 925 (1969).

3. H. Sumitomo and J. Fujii, Bull. Chem. Soc. Japan, 43, 547 (1970).

4. R. F. Fischer and C. W. Smith, U. S. Patent 2857422 (1958); Chem. Abstr., 53, 5203 h (1959).

5. O. Vog1, J. Polym. Sci., 55, 215 (1961).

6. J. Furukawa, T. Saegusa, H. Fujii, A. Kawasaki, H. Imai, and Y. Fujii, Makromol. Chem., 37, 149 (1960).

7. H. Sumitomo, K. Kobayashi, and T. Saji, Abstracts, SPSJ 20th Annual Meeting, Tokyo, May 25, 1971, p 233.

8. M. Goodman and J. Brandrup, J. Polym. Sci., Part A, 3, 327 (1965).

9. E. G. Brame, Jr., R. S. Sudol, and O. Vogl, J. Polym. Sci., Part A, 2, 5337 (1964).

10. E. G. Brame, Jr. and O. Vogl, J. Macromol. Sci. (Chem.), A1, 277 (1967).

11. J. L. Jungnickel and C. A. Reilly, J. Mol. Spectry, 16, 135 (1965).

12. G. Natta, P. Corradini, and I. W. Bassi, $J$. Polym. Sci., 51, 505 (1961).

13. G. Wasai, T. Iwata, K. Hirano, M. Suragano, T. Saegusa, and J. Furukawa, Kogyo Kagaku Zasshi (J. Chem. Soc. Japan, Ind. Chem. Sect.), 67, 1920 (1964).

14. T. Higashimura, "Cation Jūgō," Kagakudōjin, Kyoto, 1971, p 87.

15. H. Yamaoka, T. Higashimura, and S. Okamura, Kobunshi Kagaku (Chem. High Polymers), 18, 561 (1961). 